
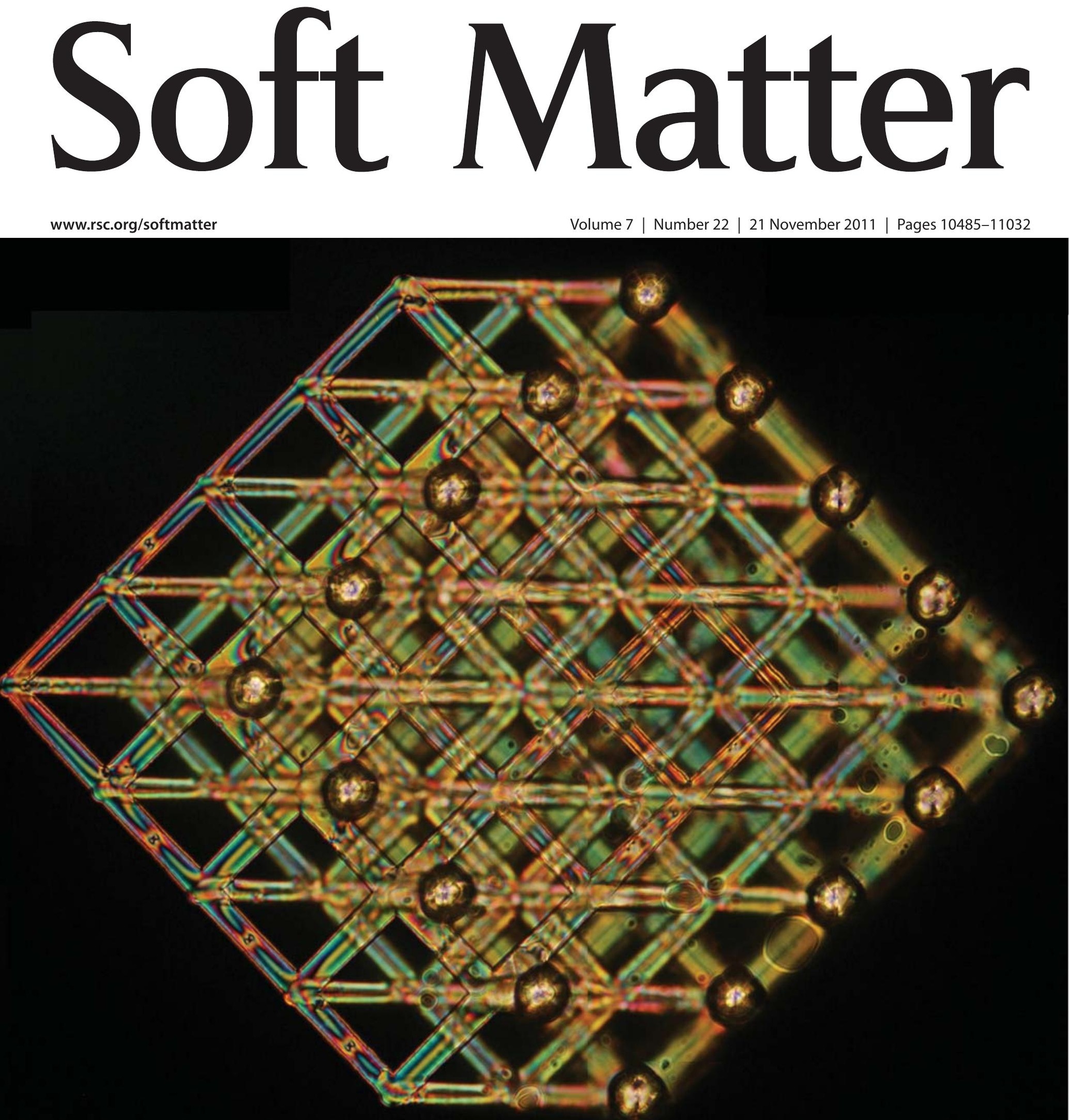


\title{
Topological defects of nematic liquid crystals confined in porous networks $\dagger$
}

\author{
Francesca Serra, ${ }^{* a}$ Krishna C. Vishnubhatla, ${ }^{b}$ Marco Buscaglia, ${ }^{a}$ Roberto Cerbino, ${ }^{a}$ Roberto Osellame, ${ }^{c}$ \\ Giulio Cerullo ${ }^{c}$ and Tommaso Bellini ${ }^{a}$
}

\author{
Received 3rd May 2011, Accepted 18th July 2011 \\ DOI: $10.1039 / \mathrm{c} 1 \mathrm{sm} 05813 \mathrm{~d}$
}

\begin{abstract}
We study the formation of topological defects in nematic liquid crystals confined in open bicontinuous networks produced in glass by femtosecond laser micromachining. We obtain a careful classification of the number and localisation of the defects as a function of the topological properties of the network. Our findings lead to a general formula that predicts the total topological charge in open complex networks, thus complementing the classic Stein-Gauss theorem. Our result provides a justification for the observed multistability of nematics confined in porous networks.
\end{abstract}

\section{Introduction}

Multistable devices with memory based on nematic liquid crystals (NLCs) require energy only for switching between different configurations, which are then spontaneously maintained. Therefore they have recently generated significant technological interest for applications to memories and electronic paper displays. In general, multistability arises whenever the liquid crystal is subject to frustrating boundary conditions creating multiple topologically distinct configurations that minimise the energy. ${ }^{1,2}$ These configurations can be associated with certain topological defects, i.e. discontinuities in the order parameter space, all characterised by a "topological charge" that depends on the arrangement of the nematic director in their proximity. ${ }^{3-6}$ Configurations become metastable when the position of the topological defects is constrained by boundary conditions: this is the case, for example, of the tristable nematic device. ${ }^{7}$ A very recent study by Araki et al. has shown that in bicontinuous networks with homeotropic boundary conditions the nematic ordering induced by external fields is maintained whenever the geometry of the network is able to stabilise the trajectories of the defect lines. ${ }^{8}$ This result provides a basis for understanding previously observed memory properties in NLCs,,${ }^{9,10}$ and it also shows the importance of the rational design of materials with specific and tunable memory properties. The topological properties of the networks can be defined by means of computer simulations, which are, however, expensive and time consuming. We therefore looked for a simpler method to evaluate the total

${ }^{a}$ Dipartimento di Chimica, Biochimica e Biotecnologie per la Medicina, Università di Milano, Via F.lli Cervi 93, 20090 Segrate, MI, Italy. E-mail: francesca.serra@unimi.it

${ }^{b}$ Center for Nano Science and Technology of IIT@POLIMI, Via Pascoli 70/3, 20133 Milano, Italy

'Istituto di Fotonica e Nanotecnologie, CNR - Dipartimento di Fisica, Politecnico di Milano, Piazza Leonardo da Vinci 32, 20133 Milano, Italy $\dagger$ Electronic supplementary information (ESI) available. See DOI: $10.1039 / \mathrm{c} 1 \mathrm{sm} 05813 \mathrm{~d}$ topological charge of a generic 3D network, which can allow rapid and reliable prediction of its multistability properties. In simple cases, computing the topological charge is effectively equal to evaluating the total number of topological defects.

The classic theorem that is invoked in the case of NLCs with homeotropic (perpendicular) anchoring was formulated by Stein in $1979^{11}$ and we will call it the Stein-Gauss (SG) theorem. The theorem relates the total topological charge of confined NLCs to the Euler characteristic of the confining surface. However, this result cannot be used, for example, for the system analysed in Araki et al.: ${ }^{8}$ first, the theorem only applies to NLCs incorporated in smooth closed surfaces; in addition, it is valid for geometries where only point defects or reducible loops (RLs), i.e. loops contractible to a point, are stable. In this paper, through a combination of experimental results and theoretical arguments, we formulate a simple expression enabling calculation of the topological charge in open networks that are beyond the reach of the validity of the SG theorem.

\section{Results and discussion}

We decided to take a heuristic approach and work on a model experimental system. We chose to start working on a network that is open and bicontinous, but where the geometry and the size of the interconnecting channels are such that point defects are the only type of topological defects observed. The network was produced in fused silica using femtosecond laser microfabrication. This technique ${ }^{12,13}$ consists of laser irradiation in the volume of the substrate followed by chemical etching in HF solution selectively removing the irradiated material. The advantages of this method are that: (i) it creates directly buried microchannels; and (ii) it produces intrinsically circular channel cross-sections. This last feature is particularly interesting for the present work since it allows the model developed for the analysis of NLC alignment in capillaries to be directly applied. ${ }^{14}$ With this tool we could produce 3D bicontinuous networks, with tens of micron-size channels, as shown in Fig. 1a-b. 


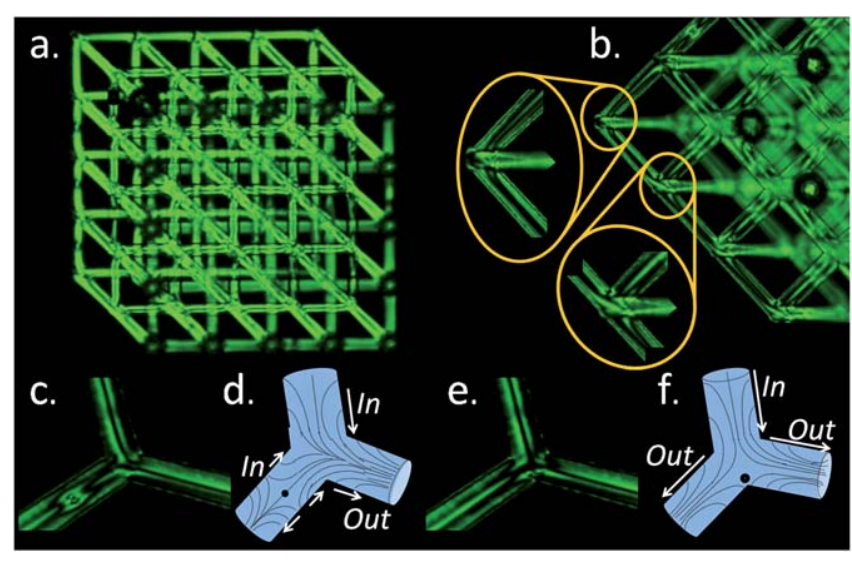

Fig. 1 Microfabricated glass channels filled with NLCs viewed between crossed polarisers. (a) Channels arranged in a skewed cubic structure (focus on the middle plane). (b) Detail and zoom on two nodes with 3 and 4 arms. Polarisers are at $45^{\circ}$ with respect to the channels axis. (c-f) $2 \mathrm{D}$ analogues of a 3-arm node with a point defect first along the channel (c) then in the centre of the node (e). (d) and (f) are pictorial representations of the nematic director in the observed area. Arrows indicate the escape radial (ER) In or Out in the channels.

Such a complex structure can be greatly simplified by considering it as a "jigsaw" made of building blocks consisting of a certain number of channels all merging in one node, as shown, for example, in Fig. 1b. In the figure, two nodes are highlighted, respectively with 3 and 4 arms. We call the number of arms the valence $v$ of the node. We hence focussed on simpler, 2D structures, made by single nodes with $v=3$ (Fig. 1c-f), 4,5 or 6 (all the possible valences found in the cube in Fig. 1) where we were able to precisely count the topological defects.

The channels were filled with the thermotropic NLC CCN47 by Merck. The low birefringence of this compound allows the analysis of its conformation under crossed polarisers even in thick channels, using quasi-monochromatic illumination. ${ }^{15}$ For every observation, the NLC was heated above the nematicisotropic transition temperature and then slowly cooled down into the nematic phase. We saw that along the channels the NLC adopted the escape radial (ER) configuration with point defects: ${ }^{14,16,17}$ this is expected, as the ER configuration is preferred to the planar polar configuration, with two parallel defect lines, when the channel diameter is large (typically, more than a micron). In the ER configuration, the nematic director progressively aligns parallel to the channel axis upon moving away from the surface. This is visualised under crossed polarisers as a characteristic pattern of light and shadow (due to the birefringence of the NLC) that depends on the relative orientation of the channel axis and the polarisers. A direction of the ER with respect to the node can be assigned from the study of the birefringence pattern of the sample under crossed polarisers when the channel axis is tilted by an angle of about $22^{\circ}$ with respect to the transmission axis of one of the polarisers (this method is explained in detail in the Supplementary Materials, Section $2 \dagger$ ). We classify as In the direction of the ER when it is pointing towards the node and as Out the opposite escape direction. $A$ priori, there is no way to predict the ER direction in the channels, as the two directions are energetically equivalent.
The topological charge in the node was determined by looking at the birefringence pattern at the crossing point and also by following the dynamics of the topological defects during the equilibration time. Upon repeated thermal cycles to the isotropic phase and back, we observed a variety of topological charges in the nodes. We made at least 10 independent observations of the NLC in each of the 3-, 4-, 5- and 6-arm nodes, finding that the topological charge in the nodes always correlated to the direction of the escape of the channels around it.

As examples, we show and explain here some of the director patterns in the 3-and 4-arm nodes. Fig. 1c and 1e show two different transmission patterns through cross polarisers of a 3-arm node. Their analysis leads to the reconstruction of the director pattern as in Fig. 1d and 1f. The node is defect-free and, in the immediate proximity of the node, two channels have an In escape and one has an Out escape (Fig. 1c,d). A hyperbolic point defect is present in one of the arms, appearing as a "butterfly"like pattern. As the defect travels to the node and localises at one corner (Fig. 1e,f), we obtain a different condition: the defect in between the two Out channels, escape directions being one In and two Out.

Fig. 2 shows some of the experimental observations in a 4-arm node. The structure is defectless when one arm is Out while the other three are In (panels a, b, c). We instead find one hyperbolic hedgehog when two channels are Out and two are In (panels d, e, f). One radial hedgehog is found when all channels are In (panels $\mathrm{g}, \mathrm{h}, \mathrm{i}$ ). The results of the observations of defects for 3-, 4-, 5- and 6 -arm nodes are summarised in the first four columns of Table 1, where every configuration is classified on the basis of the number $n_{\text {Out }}$ of channels with an Out escape.

It is important to clarify here the notation we have adopted for the topological charge. Hyperbolic and radial point defects in nematics are topologically equivalent (they can be transformed
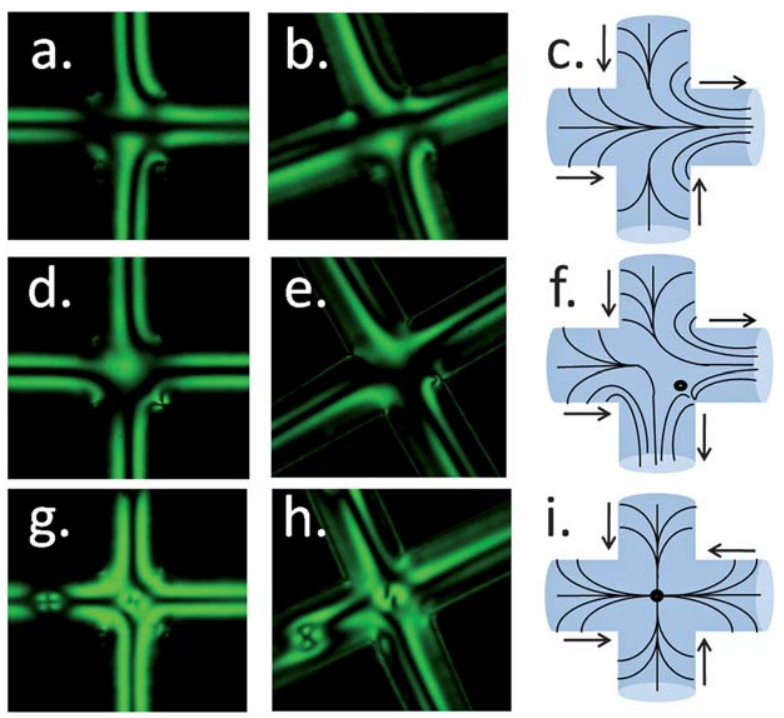

Fig. 2 NLC in 4-arm nodes. (a) 1 Out configuration (no defects in the node); (d) 2 Out with one hyperbolic hedgehog. (g) 4 In (radial hedgehog in the node). (b, e, h) Channels form an angle around $20^{\circ}$ with respect to the axis of the crossed polarisers. (c, f, i) Representations of the nematic director configuration inside the channels. Arrows indicate the In and Out directions. Defects are highlighted with black dots. 
Table 1 Total charge $M$ of topological defects observed in nodes with 36 arms and in a roundabout (last column), linked to the number of Out channels. Empty table entries are for configurations which were not clearly observed experimentally

\begin{tabular}{lrrrrr}
\hline$n_{\text {Out }}$ & 3-arm & 4-arm & 5-arm & 6-arm & Round \\
\hline $\mathbf{0}$ & & +1 & & & 0 \\
$\mathbf{1}$ & 0 & 0 & & 0 & -1 \\
$\mathbf{2}$ & -1 & -1 & -1 & -1 & -2 \\
$\mathbf{3}$ & -2 & -2 & -2 & & -3 \\
$\mathbf{4}$ & & & & & -4 \\
\hline
\end{tabular}

continuously into one another); therefore, assigning a positive or negative topological charge to them is arbitrary. ${ }^{18,19}$ Nevertheless, it is very common to assign a positive charge +1 to a radial point defect and a negative charge -1 to a hyperbolic hedgehog, ${ }^{5,20}$ according to the so-called winding number. The reason is that a radial point defect and a hyperbolic defect, when brought together, annihilate and give a defect-free state. In the presence of a line defect, however, the situation is much more complex and the topological charge of the final state depends on the coalescence path of the defects. Here, however, we are observing only point defects with unit topological charge (energetically much more favorable than point defects with a higher integer charge) which are stable after an equilibration time and which are separate in space. We can therefore assume that the radial point defects and the hyperbolic point defects do not change their winding number in this context: so, we will follow the notation in ref. 5 and 16 which assigns a +1 charge to the radial point defects and a -1 charge to the hyperbolic point defects.

The topological charge $M$ in the nodes is found, from our experiments, to follow the simple rule:

$$
M=1-n_{\text {Out }}
$$

where $n_{\text {Out }}$ indicates the number of the channels with an Out ER direction.

With these nodes being intrinsically open structures, the SG theorem cannot be rigorously applied. However, we show now that this empirical formula is coherent with it. The SG theorem states that, in a confined NLC with homeotropic anchoring, the total topological charge $M$, which is the sum of the topological charges $m_{i}$ of individual point defects, equals the Euler characteristic $E$ of the confining surface divided by $2: M=\Sigma_{i} m_{i}=1 / 2 E$. $E$ is the integral of the Gaussian curvature over the whole surface and it is invariant for all the topologically equivalent surfaces, i.e. surfaces that can be mapped onto each other with a continuous invertible function. In a simple case such as a sphere, $E=2$, therefore $M=1$, corresponding to a radial hedgehog or a reducible loop with charge +1 in the centre of the sphere as shown in Fig. 3a. A sphere is also topologically equivalent to a closed cylinder (with homeotropic anchoring everywhere), therefore the expected topological charge is +1 . If one wants to have an ER configuration in the middle of the cylinder the defect is pushed towards one "cap" of the cylinder, as shown in Fig. 3a. The same concept of homeotropic capping can then be applied to all the channels merging in a node: doing this, one sees that the In channels can be smoothly capped with no topological defects, but when an Out channel is capped, either a point or a ring defect

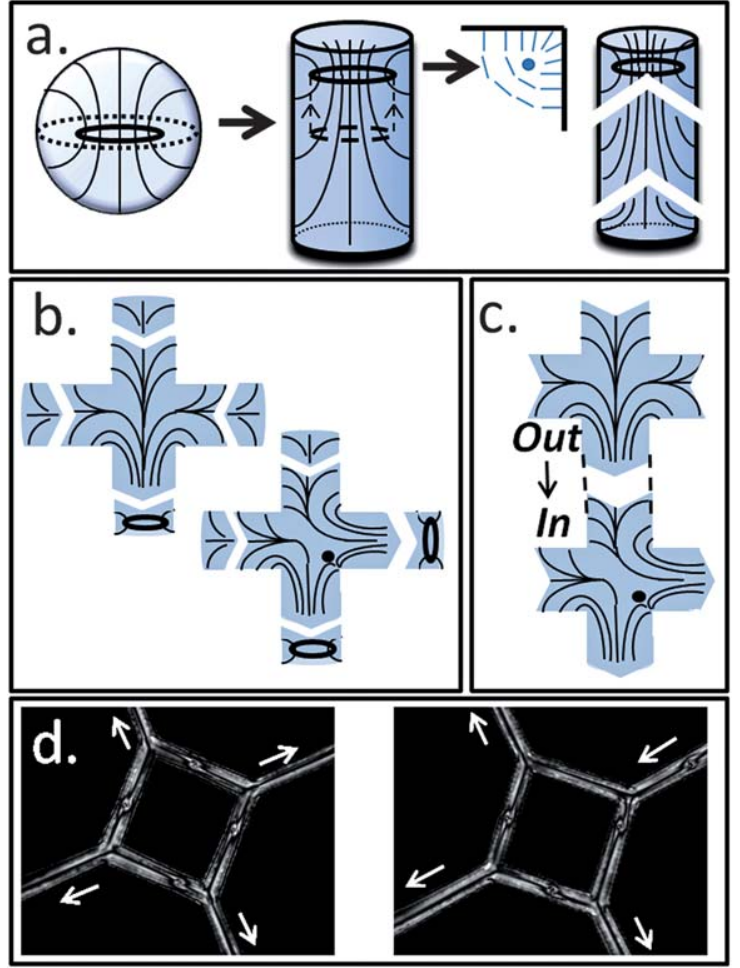

Fig. 3 Topological defects of NLCs in microchannels. (a) Pictorial image of a sphere and a capped cylinder with the internal defect. On the right, the cylinder is ideally divided into 3 parts, where the defect is found only in the cap. (b) Pictorial representation of two possible configurations of the nematic director in a 4-arm node (analogous to those in Fig. 2c,2f) and the ideal "capping". (c) How to join two adjacent nodes (scheme). (d) Roundabout type structure filled with NLCs and different escape directions, indicated by arrows.

with +1 charge is necessarily created. Hence, the capping brings about an additional topological charge of $m=+1$ for each of the Out channels (Fig. 3b). The "node + channels" structure, when capped, must also have total topological charge $M=+1$, being topologically equivalent to a sphere. Thus, in order to determine the topological charge contained inside the open node, one must subtract the charge carried away by the channels with an Out escape, and eqn (1) follows.

To approach the analysis of the 3D network shown in Fig. 1a, it is useful to show in a simpler structure that the topology of the systems plays an important role in determining the topological charge. We observed the arrangement of NLCs in a 2D "roundabout"-type structure, shown in Fig. 3d. Here we only show two configurations where all defects are localised in the channels of the circuit, and not in the nodes, so that they are very easily visible. If one assumes that $n_{\text {Out }}$ only refers to the external channels, i.e. channels connecting the network to the outer space, it would be tempting to think that the whole structure will behave as a 4-arm node. This is contradicted by the experimental observation, reported in the last column of Table 1, which shows that $M=-n_{\text {Out }}$. The presence of a hole in the middle of the node is therefore relevant for the topological charge. A successful approach to circumvent this problem is to consider the whole structure as four 3-arm nodes joined together and to add up the charge of the single nodes. 
We counted and added up the point defects in the 3D network in Fig. 1a. A fraction of them are along the channels and they are clearly detectable through the birefringence pattern, while some of them, located in the nodes, are not. However, by observing each node separately and counting, for each node, the number of In and Out channels, it is possible to assign every node a charge, by using eqn (1). Four different observations in the network shown in Fig. 1 (obtained by heating to isotropic and cooling into the nematic phase and corresponding to different arrangements of the topological defects) gave a computed total topological charge $M=-95 \pm 1$. The uncertainty is inevitable in this case, as it is not possible to determine, in this system, the In or Out direction of the external channels connecting the network to the glass surface.

We highlight that in these simple interconnected systems, like the $3 \mathrm{D}$ structure and the roundabout, the configurations can be stable over several days. These systems already show multistability: by applying an electric field or a mechanical stress (as a gentle pressure on the external holes of the microchannels) some defects change their position and move about the structure, while the In and Out escape can be permanently changed (an example is shown in Supplementary Materials, Section 3†).

In many cases, it is not possible to determine the ER direction for each node as we have just done: for example, porous networks can be opaque and not accessible to the observation. A simpler method to evaluate the topological charge of a network, which does not require a detailed observation of each node, relies on eqn (1). To this aim, we use the basic jigsaw rule that a defectless channel connecting two nodes is necessarily In for a node and Out for the adjacent node, as shown in Fig. 3c. If a channel contains a point defect, the defect can be effectively assigned to one node or to the adjacent one, the ER direction after the defect being the only relevant information. With these considerations, it is clear that, where $v$ is the valence of the nodes, the average number of channels escaping Out from each node is $v / 2$. Then, eqn (1) can be applied and the total topological charge is given by the sum of the topological charges of all the nodes. A general expression for the topological charge becomes:

$$
M=\sum_{i=1}^{N}\left(1-\nu_{i} / 2\right)-\Delta_{\mathrm{s}}=N(1-<\nu>/ 2)-\Delta_{\mathrm{s}} .
$$

Here $N$ is the total number of nodes, and $\langle v\rangle$ is the average valence of the nodes. $\Delta_{\mathrm{s}}$ is a term that takes into account the escape of the external channels, i.e. channels that do not connect nodes: $\Delta_{\mathrm{s}}=n_{\text {Out }}-N_{\text {ext }} / 2$ where $N_{\text {ext }}$ is the number of external channels, among which $n_{\text {Out }}$ escape outwards. While $\Delta_{\mathrm{s}}$ is crucial in determining the topological charge of small structures, it becomes negligible in large systems, or in simulated systems having periodic boundary conditions. Eqn (2) accounts for the topological charge of the roundabout shown in Fig. 3d; it also provides an estimation of the topological charge of the 3D network in Fig. 1, calculating $M=-95$, in agreement with the observation.

Eqn (2) allows estimating the amount of topological charge that must be present in networks of various symmetry. The results are reported in Table 2. A periodic bicontinuous medium can be thought of as a lattice made of intersecting channels, organised in repeated unit cells with known symmetry.
Table 2 Unit cell types (C: cubic; BCC: body-centered cubic; FCC: facecentered cubic; P: porous medium), with the valence $v$ of the nodes and the topological charge per cell $M_{\mathrm{c}}$.

\begin{tabular}{lllll}
\hline Unit cell & C & BCC & FCC & P \\
\hline$v$ & 6 & 4 & 12 & 3 \\
$M_{\text {c. }}$ & -2 & -2 & -5 & -1 \\
\hline
\end{tabular}

Considering a system of packed spheres, the interstices, or pores, between spheres can be filled with NLCs, thus forming a bicontinuous medium.

The simplest example is the cubic lattice. The unit cell is formed by one sphere, or, equivalently, by one pore. The topological charge per pore (or node) $m$ is equal to $m=(1-v / 2)$. In a cubic lattice, $v=6$, therefore the topological charge per cell $\left(M_{\mathrm{c}}\right)$ is $M_{\mathrm{c}}=-2$.

In a body centered cubic (bcc) lattice, every sphere touches 8 other spheres. Every pore connects 4 other pores $(v=4)$ and there are 2 pores per unit cell. Thus $M_{\mathrm{c}}=2(1-4 / 2)=-2$.

The interstitial sites of am fcc lattice of close packed spheres have the peculiar shape of a cuboctahedron joined with two octahedra (a clear view of the geometry of the interstitial site is shown in ref. 21). The number of "escapes" from such an interstitial site, corresponding to the number of arms of the node, is 12, and thus: $M_{\mathrm{c}}=(1-12 / 2)=-5$.

Many of the porous matrices experimentally considered as incorporating hosts for NLCs have irregular geometries, thus making it impossible to give a general role for their topological charge. However, a large number of them, such as the membrane filters, are obtained by spinodal decomposition, a process whose topology has been investigated. ${ }^{22}$ In this case, the average number of nodes per domain (a sort of repeated unit) is 2 and the average valence is $v=3$, therefore $M_{\mathrm{c}}=-1$. This agrees with the notion that the porous surface obtained from the spinodal decomposition is similar, to a certain extent, to a Schoen's gyroid, which has the same topological features.

So far, we have restricted our attention to point defects or reducible loops. In porous materials where the size of the channels is smaller or the ratio between the mean pore length and mean solid length is larger, irreducible loops (ILs) normally appear, as reported in ref. 8. ILs embrace the solid portions of the bicontinuous matrices and carry topological charge, a situation very far away from the range of validity of the SG theorem. Among the geometries studied in ref. 8, nematics with homeotropic anchoring in a cubic bicontinuous network (the one exhibiting more memory) display alternating parallel planes of ILs and RLs. In a uniform strong field, the topological charge of each loop, irreducible or not, is - 1 (in analogy with Saturn-rings around spherical particles ${ }^{23}$ ). Thus, eqn (2) agrees with the computer simulations in ref. 8, which show one IL and one RL per node. This successful comparison suggests that eqn (2) accounts for the total number of defects in a system with ILs, where the SG theorem could not make predictions.

We want to suggest a possible, intuitive explanation of why in this case ILs contribute equally to RLs to the total charge. For this we rely on the works by Ravnik et al. ${ }^{24}$ and by Čopar and Žumer, ${ }^{25}$ which show and classify all variety of loops entangling spherical colloids immersed in NLCs. What is most relevant for our work is that they prove that the Saturn rings surrounding two colloids can 
also form a so-called "entangled hyperbolic defect", or $\theta$-structure: a big loop encircles the two colloids forming a smaller reducible loop in between. This can be extended to a case of an array of pillars: the configuration with one IL per pillar is replaced by a condition where only one IL is left (less and less relevant for large systems) while the others are turned into RLs, thus recovering the conditions where eqn (2) is valid.

It is worth noticing that the spatial arrangement of ILs within a porous network has to obey topological constraints. In Fig. 4a a bicontinuous structure is sketched. The aperture area between adjacent pillars (such as the pink shadowed region in Fig. 4) can be regarded as a short channel. In every such channel, either none or two disclination lines can be found, corresponding to the ER or the planar polar configuration, respectively. Hence, as one encircles a pillar of the structure with an IL (green line), this needs to be bordered by one other IL in every channel it runs through, while a third IL - dashed red line - is inadmissible. As a consequence, ILs in 3D bicontinuous networks are arranged so to define self-avoiding surfaces made of loops paired through channels. In the cubic lattice, there are no possible ways of placing enough ILs to reach the topological charge calculated with eqn (2), equivalent to two ILs per node: this is a simple explanation of why in the computer simulations ${ }^{8}$ half the topological charge is carried by the RLs. The arrangement of ILs in self-avoiding surfaces and of RLs enables us to appreciate the large degeneracy that characterises nematics in bicontinuous networks: the possible distributions of ILs and point defects are numerous, and their estimation is a challenging task. ${ }^{26}$ This concept lays at the basis of the multistability.

In conclusion, on the basis of experimental observation and theoretical arguments we have developed a tool to compute the total topological charge in an open network with arbitrary connectivity, where the classic Stein-Gauss theorem is not applicable. We tested this method on simple experimentally accessible networks made of laser microfabricated glass channels and we found that our predictions were verified in simulated systems containing irreducible loops.

The authors would like to thank Cariplo foundation (grant 2008-2413) and T. Araki, F. Bisi, S. Copar, F. Giavazzi, H. Tanaka, E. Terentjev, E. Virga for useful discussion.

\section{Experimental}

The fabrication of microchannels by femtosecond laser irradiation followed by chemical etching (FLICE) is a powerful
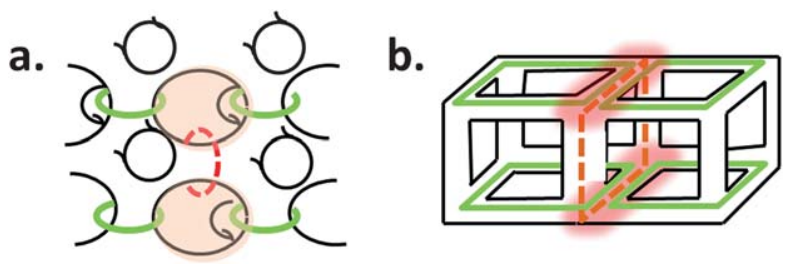

Fig. 4 ILs in bicontinuous cubic: (a) a portion of a bicontinuous cubic lattice and (b) its schematic structure with interconnected channels. While the highlighted ILs (thick lines, green online) are allowed, the IL in dashed line (red online) cannot be placed as in the figure, because there would be two channels with three defect lines (red shading online). technique that has the following main advantages: (a) it is a maskless technique and hence allows rapid prototyping of novel configurations; (b) it creates directly buried microchannels, without the need for sealing with a cover glass; and (c) it is a three-dimensional technology, thus allowing arbitrary designs to be fabricated in 3-D (see online Supplementary Materials, Section 1, for further details $\dagger$ ). The FLICE technique applied to fused silica consists of two steps: (1) irradiation of the sample with focussed femtosecond laser pulses; and (2) etching of the laser modified zone by a hydrofluoric acid (HF) solution in water. For the irradiation of the material we use the second harmonic $(515 \mathrm{~nm})$ of a home built, cavity-dumped Yb:KYW oscillator providing 350-fs laser pulses at repetition rates up to 1 $\mathrm{MHz} .{ }^{13}$ The irradiated patterns are produced using 300-350 nJ pulse energy, circular polarization, $1 \mathrm{MHz}$ repetition rate, 0.6 NA microscope objective (focussing to a beam diameter of about $1 \mu \mathrm{m}$ at a depth of $400 \mu \mathrm{m}$ below the surface) and $1 \mathrm{~mm} \mathrm{~s}^{-1}$ translation speed. The subsequent chemical etching is performed by immersing the irradiated sample in an ultrasonic bath of $20 \%$ HF solution in water for $4 \mathrm{~h} .{ }^{12}$ The chemical etching selectively removes the irradiated paths creating directly buried microchannels with circular cross-section. In order to start the chemical etching of the buried structure, the irradiated path has $90^{\circ}$ bends at the end of each channel thus reaching the top surface, where the acid can start to attack. After the etching process, the portions of microchannels connecting the buried structure to the top surface act as access holes and allow the filling of the microfluidic network with liquid crystals. Different geometries of the channel network have been explored in the experiments presented in this paper; several junctions have been fabricated with a different number of crossing arms, i.e. 6, 5 and 3. The microchannel shape is not cylindrical but conical. This is a well known feature of microchannels fabricated by the FLICE technique, ${ }^{12}$ which can be compensated if needed. ${ }^{13}$ However, in the present application this feature is advantageous since we wanted small channels in the junction (in the order of 30-50 $\mu \mathrm{m}$ diameter) to easily localise the defects, but reasonably large access holes (about $300 \mu \mathrm{m}$ diameter) to easily fill them and reduce the risk of clogging the channels. The 3D tapering of the channel to satisfy these two requirements was automatically achieved with the FLICE technique. We also exploited the capability of the FLICE technique of creating structures in $3 \mathrm{D}$. The structure we built is a skewed cube: it consists of 3 layers in depth, each separated from the other by $200 \mu \mathrm{m}$ and the shallowest one buried $200 \mu \mathrm{m}$ below the top surface of the sample. Each layer consists of a square grid with $200 \mu \mathrm{m} \times 200 \mu \mathrm{m}$ openings. In order to improve the visualization of the structure when looking for point defects along the channels we decided to skew the cube horizontally by $250 \mu \mathrm{m}$ along the diagonal of the cube base. Each node of the grid is then connected to the corresponding one in the layer above/below by a tilted microchannel, which can be inspected from a top view. The fabrication of the $3 \mathrm{D}$ structures is performed with the same technology used for the 2D structures discussed above. In particular, to facilitate the uniform HF etching of the irradiated 3D structure, the bottom layer of the structure is connected by 16 access holes to the top surface, where the acid can start to penetrate. The structure is etched for $45 \mathrm{~min}$ and this creates microchannels of $\sim 40 \mu \mathrm{m}$ diameter. More details and pictures can be found in the Supplementary Material. $\uparrow$ 
The channels were filled with the thermotropic LC 40-transbutyl-4-cyano-4-heptyl bicyclohexane (CCN47) purchased from Merck. CCN47 was chosen as the NLC because of its low birefringence, about $1 / 10$ th of that of cyanobiphenyl compounds, commonly used in display applications; in fact, a high birefringence LC is difficult to observe if the channel radius is larger than a few microns. $\mathrm{CCN} 47$ has a nematicisotropic (NI) phase transition at $T_{\mathrm{NI}}=59{ }^{\circ} \mathrm{C}$, a smecticnematic phase transition at $T_{\mathrm{SN}}=30^{\circ} \mathrm{C}$. Below this temperature the material is solid-like.

LC samples were observed with a Nikon Optifot2-pol polarising microscope equipped with a Nikon DS-5M CCD camera. Objectives had a magnification of $\times 10, \times 20$ or $\times 40$. The polariser was fixed while the analyser could be rotated up to $180^{\circ}$. The sample was mounted on a custom-made, computer-controlled heater, and the heater was placed on a rotating stage equipped with a goniometer with a precision of $1^{\circ}$. For the observation of liquid crystals, we chose to use green light, filtered between 530 and $570 \mathrm{~nm}$. In fact, on the one hand, using monochromatic light is useful for the interpretation of data and quantitative analysis; on the other hand, the human eye is more sensitive to green light and the sample features could be better distinguished.

As many NLCs, CCN47 spontaneously adopts a planar alignment on glass, i.e. with the nematic director randomly oriented in directions parallel to the glass wall. In order to avoid this alignment in the channels and to have instead normal (homeotropic) anchoring at the channel walls, the surfactant DDAB (didodecyldimethylammonium bromide, purchased from Sigma-Aldrich) was dissolved in CCN47. As such, the CCN47DDAB mixture is placed in contact with the glass wall, the hydrophilic head of DDAB adheres to the glass walls, thus forming a surfactant monolayer which provides homeotropic anchoring. Various tests were conducted at different concentrations of DDAB and the best anchoring was obtained for a weight concentration of $0.1 \%$. Channels were filled by bringing the CCN47-DDAB mixture to temperatures above the NI transition, and then by placing a drop close to one channel pit. The NLC filled the channel spontaneously. In case bubbles appeared in the channels, the samples were kept under vacuum for a few hours. Samples were left to anneal above the nematic-isotropic transition for several minutes, then cooled down to about $55^{\circ} \mathrm{C}$ with a cooling rate ranging from 0.2 to $5{ }^{\circ} \mathrm{C} \mathrm{min}^{-1}$.

Several measurements were thus performed on each of the microstructures, either by annealing and cooling down several times or by washing away the liquid crystal and re-filling. Three samples with a 3-arm node were fabricated. The samples did not show any significant difference from the point of view of the NLC behaviour. Sixteen measurements were performed and the variety of behaviours could be classified in a few configurations, as described in the paper. Analogously, 25 measurements were performed on 4-arm nodes (two different samples), 13 measurements on 5-arm nodes (two different samples) and 19 measurements on 6-arm nodes (three different samples). In the latter two cases, not all the possible combinations of In and Out channels were observed. On the single roundabout sample, we performed 31 observations.

\section{References}

1 P. Brett, A. Graham, J. C. Jones, E. L. Wood, G. P. Bryan-Brown and J. R. Hughes, SID Symposium Digest, 2000, 31, 124.

2 A. Majumdar, C. J. P. Newton, J. M. Robbins and M. Zyskin, Phys. Rev. E, 2007, 75, 051703 .

3 P. G. D. Gennes and J. Prost, The Physics of Liquid Crystals, 2nd edn, Oxford Science Publications, 1995.

4 M. Kleman, Rep. Prog. Phys., 1989, 52, 555.

5 S. Chandrasekhar and G. S. Ranganath, Adv. Phys., 1986, 35, 507.

6 M. Kleman and O. Lavrentovich, Philos. Mag., 2006, 86, 4117-4137.

7 J. H. Kim, M. Yoneya and H. Yokohama, Nature, 2002, 420, 159.

8 T. Araki, M. Buscaglia, T. Bellini and H. Tanaka, Nat. Mater., 2011, 10, 303.

9 T. Bellini, M. Buscaglia, C. Chiccoli, F. Mantegazza, P. Pasini and C. Zannoni, Phys. Rev. Lett., 2002, 88, 245506.

10 M. Rotunno, M. Buscaglia, C. Chiccoli, F. Mantegazza, P. Pasini, T. Bellini and C. Zannoni, Phys. Rev. Lett., 2005, 94, 097802.

11 D. L. Stein, Phys. Rev. A, 1979, 19, 1708.

12 V. Maselli, R. Osellame, G. Cerullo, R. Ramponi, P. Laporta, L. Magagnin and P. L. Cavallotti, Appl. Phys. Lett., 2006, 88, 191107.

13 K. C. Vishnubhatla, N. Bellini, R. Ramponi, G. Cerullo and R. Osellame, Opt. Express, 2009, 17, 8685-8695.

14 G. G. Peroli and E. Virga, Phys. Rev. E, 1996, 54, 5235.

15 M. Buscaglia, G. Lombardo, L. Cavalli, R. Barbieri and T. Bellini, Soft Matter, 2010, 6, 5434 .

16 G. P. Crawford, D. W. Allender and J. W. Doane, Phys. Rev. A, 1992, 45, 8693.

17 G. D. Luca and A. D. Rey, J. Chem. Phys., 2007, 127, 104902.

18 K. Jaenich, Acta Appl. Math., 1987, 8, 65.

19 N. D. Mermin, Rev. Mod. Phys., 1979, 51, 591.

20 A. M. Sonnet and E. G. Virga, Liq. Cryst., 2010, 37, 785.

21 D. Kang, A. A. Z. J. E. Maclennan, N. A. Clark and R. H. Baughman, Phys. Rev. Lett., 2001, 86, 4052.

22 H. Jinnai, H. Watashiba, T. Kajihara and M. Takahashi, J. Chem. Phys., 2003, 119, 7554-7559.

23 E. Terentjev, Phys. Rev. E, 1995, 51, 1330.

24 M. Ravnik, M. Skarabot, S. Žumer, U. Tkaleč, I. Poberaj, D. Babic, N. Osterman and I. Musevič, Phys. Rev. Lett., 2007, 99, 247801. 25 S. Čopar and S. Žumer, Phys. Rev. Lett., 2011, 106, 177801.

26 U. Glaus, Phys. Rev. Lett., 1986, 56, 1996. 